\title{
Radical cystectomy is the treatment of choice for invasive bladder
}

\section{cancer}

\author{
Faysal A. Yafi, MD; Wassim Kassouf, MD, FRCSC
}

$\mathrm{B}$ ladder cancer is the second most common urologic cancer and the sixth most common overall cancer in Canada, accounting for 6700 new cases per year. It has the fourth highest incidence among cancers in men and the twelfth in females. It represents up to $5.8 \%$ of new cancer cases and $3.3 \%$ of deaths in Canadian men. ${ }^{1}$ Tobacco smoking is the most established risk factor, leading to $50 \%$ to $65 \%$ and $20 \%$ to $30 \%$ of male and female cases, respectively. ${ }^{2}$ Other risk factors include occupational exposure to benzene derivatives and aryl amines, phenacetin abuse, external beam radiation therapy, dietary factors, cyclophosphamide and chronic urinary tract infections (especially with schistosomiasis in endemic areas). ${ }^{3-8}$

\section{Radical cystectomy for muscle-invasive bladder cancer}

For muscle-invasive tumours, radical cystectomy with pelvic lymphadenectomy has been the gold standard for the past several decades. New advancements in neoadjuvant and adjuvant chemotherapy, radiation therapy and bladderpreservation protocols have been introduced for a subset of patients. In the past, radical cystectomy was not universally accepted because of high recurrence, complication rates and poor subsequent quality of life. However, many improvements have been made in the surgical approach and techniques. Complication and perioperative mortality rates have been declining, with reported rates as low as $17 \%$ to $32 \%$ and $2 \%$ to $3 \%$, respectively. ${ }^{9-11}$ Other voiced concerns with surgery were incontinence and impotence. With an orthotopic ileal neobladder, daytime and nocturnal continence rates as high as $85 \%$ to $90 \%$ and $50 \%$ to $90 \%$, respectively, can be achieved. ${ }^{12}$ Also, with unilateral and bilateral nerve-sparing cystectomy, potency rates of $33 \%$ and $60 \%$ can be achieved. ${ }^{13}$ These results are comparable to radiation therapy, with $54 \%$ of these patients having adequate erections. ${ }^{14}$

There is also recent interest on the importance of lymphadenectomy and its therapeutic and prognostic value. About $25 \%$ of patients present with lymph node involvement at the time of surgery. Skinner was among the first physicians to advocate radical cystectomy with wider resection of perivesical soft tissue and extended lymph node den- sity (LND) in an effort to decrease local recurrence of disease and improve cure rates for invasive bladder cancer. ${ }^{15}$ A combined effort between the Cleveland Clinic and the University of Bern compared 2 groups of patients who underwent a radical cystectomy with a limited LND in the prior and an extended LND in the latter and showed improved survival in the second group. ${ }^{16}$ Multiple studies show that an extended lymphadenectomy up to the aortic bifurcation offers improved patient survival with low associated morbidity and mortality. ${ }^{9-11}$ Several studies demonstrate that the number of nodes resected was associated with improved survival in patients with invasive bladder cancer. ${ }^{17-19}$ Furthermore, in the widely quoted South West Oncology Group (SWOG) trial evaluating the role of neoadjuvant chemotherapy, Herr and colleagues examined surgical factors as surrogates for outcome. ${ }^{20}$ The number of nodes removed $(>10)$ in both node-negative and node-positive patients was associated with prolonged survival and remained an independent prognostic factor even after adjusting for the use of neoadjuvant chemotherapy, surgical margin status, pT stage and nodal status. With radiation therapy, however, disease control in the pelvis may be suboptimal, as areas covered may include the internal and external iliac lymph nodes but not the aortic bifurcation where the associated toxicity can be much higher. ${ }^{21}$

More importantly, patients who are found to have occult nodal metastasis or extravesical disease at cystectomy are at high risk of recurrence and are commonly offered adjuvant chemotherapy. The benefit of adjuvant chemotherapy for pT3 or $\mathrm{pN}+$ disease has been shown in 3 randomized trials, which demonstrated significantly improved 5-year recurrence-free survival of $22 \%$ to $51 \%$ and confirmed by a meta-analysis that showed a $25 \%$ relative reduction in

The purpose of the Point/Counterpoint section is to encourage vigorous and informed discussion on controversial issues in urology through the presentation of diverse opinions. We aim for a dispassionate discussion of controversies, recognizing that strong passions may exist in support of some positions. 
the risk of death. ${ }^{22-25}$ Concomitant pelvic lymphadenectomy with radical cystectomy offers more accurate staging that increases detection of micrometastasis allowing adjuvant chemotherapy to be offered early where the tumour burden is lowest. Patients treated with bladder preservation strategies, however, are offered radiosensitizing chemotherapy, then are treated with salvage chemotherapy upon recurrence; these patients rarely receive adjuvant chemotherapy when the tumour burden is low and the chance of cure is highest. Furthermore, at our institution, many patients with muscle-invasive bladder cancer who are treated with bladder-preservation strategies often have compromised renal function, which precludes the use of sensitizing platinum-based chemotherapy in combination with radiation; these patients commonly receive gemcitabine or carboplatin, which are inferior to cisplatin for bladder cancer therapy. As such, this particular group of patients would also benefit from surgery.

In the absence of any randomized clinical phase III trials comparing radical cystectomy and bladder-preservation therapies, it is difficult to clearly delineate the optimal management option. Contemporary series show impressive 5 -year pelvic control rates of $80 \%$ to $90 \%$ and 5 -year overall and disease-specific survival (DSS) rates of $59 \%$ to $60 \%$ and $55 \%$ to $65 \%$, respectively, for radical cystectomy. ${ }^{9-11,26}$ Radiotherapy, as a single modality, is certainly suboptimal to surgery, with 5 -year overall survival (OS) of only $25 \%$ to $30 \% .{ }^{27}$ While the reported 5 -year OS rates of $48 \%$ to $63 \%$ for bladder preservation therapies consisting of transurethral resection of bladder cancer (TURBT) followed by chemoradiation may be comparable to the cystectomy group, it is noteworthy that with longer follow-up, 35\% to $45 \%$ of these patients end up requiring salvage cystectomy (due to suboptimal local disease control), which is certainly technically more challenging, with reported complications as high as $58.5 \%$ within 90 days of surgery. ${ }^{9,28-33}$ A study comparing immediate versus delayed salvage cystectomy has shown that the latter is associated with significantly higher regional lymph node metastasis (26\% vs. $12 \%)$ and shorter 5-year DSS in patients with advanced bladder cancer $(56 \%$ vs. $80 \%) .{ }^{34}$ Furthermore, in those who have their bladders preserved and choose not to undergo salvage cystectomy, the 5-year survival drops to $36 \% .{ }^{28-32}$ Ileal neobladders also become much more challenging following pelvic irradiation, with complication rates as high as $33 \%$ including prolonged urinary leakage, ureteral stenosis, fistulas, and urinary retention with a re-operation rate of $17 \%$ at 28 months and significant day and night incontinence rates of $33 \%$ and $44 \%$, respectively. 35,36

One should also not overlook the long-term risk of secondary malignancies following radiotherapy as irradiating the pelvis (e.g., in patients with prostate cancer) has been shown to increase the rate of cancer with relative risks of bladder and rectal cancers of 1.88 and 1.26 for external beam radiotherapy, 1.52 and 1.08 for brachytherapy and 1.85 and 1.21 for external beam radiotherapy-brachytherapy compared to prostatectomy alone. ${ }^{37}$ Lastly, although comparable results may be achieved in only select patients, patients with compromised renal function, hydronephrosis, concomitant carcinoma in situ (CIS) or large tumour burden will respond poorly to bladder preservation strategies with radiation. Wolf and colleagues reported that when there was CIS on initial presentation, new bladder tumour recurrences were noted in 58\% of patients following radiotherapy. ${ }^{38}$ Hydronephrosis, for its part, is associated with worse complete response rate $(37 \%$ vs. $68 \%)$, overall $(48 \%$ vs. $53 \%$ ) and DSS (55\% vs. $64 \%$ ) with combined chemoradiation and as such these patients should preferentially be candidates for surgical intervention. ${ }^{14}$ Finally, one must also take into consideration that following bladder preservation, strict follow-up with frequent and lifelong cystoscopies is required, which can be uncomfortable for patients and associated with a higher risk of bleeding and infections.

After taking into account all the above-mentioned points, chemoradiation is a good option only for select patients, such as the elderly, those with significant comorbidity or those who refuse surgery and are strongly motivated to keep their bladder while adhering to a strict surveillance regimen.

\section{Radical cystectomy for non-muscle-invasive bladder cancer}

For non-muscle-invasive tumours (NMIBC), a complete TURBT is the treatment of choice followed by intravesical therapy or close surveillance. ${ }^{39}$ These tumours should be further stratified by risk. Immediate cystectomy is advocated when there are multiple recurrent high-grade tumours, highgrade $\mathrm{T} 1$ tumours and high-grade tumours with concomitant CIS as well as in the context of Bacillus Calmette-Guerin (BCG) failure. ${ }^{39}$ At radical cystectomy, up to $43 \%$ of patients with these features have muscle-invasive disease despite treatment with intravesical therapy, lending further support to the role of early surgical intervention in this group. ${ }^{40}$ Interestingly, a delay in surgery has been shown to decrease DSS in these patients. Herr and colleagues have demonstrated that DSS is significantly longer in patients with NMIBC who recur and are subsequently treated with radical cystectomy within 2 years of BCG therapy compared to those treated after more than 2 years from BCG therapy (92\% vs. $56 \% 15$-year disease-specific survival); ${ }^{41}$ improved survival with early cystectomy was demonstrated regardless if patients with NMIBC recurred with NMIBC or with muscle inva- 
sion. In patients with high-risk NMIBC, the impact of radiation therapy has been studied in a randomized clinical trial of 210 patients with T1G3 bladder tumours comparing conservative measures and intravesical therapy to radiation therapy. No benefit was found with radiotherapy in terms of progression-free interval (hazard ratio [HR] 1.07, $p=0.785)$, progression-free survival (HR 1.35, $p=0.133)$ or OS (HR 1.32, $p=0.193) .{ }^{42}$ As such, radical cystectomy remains the primary choice in patients with high-risk NMBIC who fail BCG.

\section{Summary}

Radical cystectomy with lymphadenectomy continues to be the standard of care and primary choice for patients with muscle-invasive and recurrent or refractory NMIBC. Bladder-preservation strategies, such as chemoradiation, are good options for select patients, such as the elderly, those with significant comorbidity or those who refuse surgery and are strongly motivated to keep their bladder while adhering to a strict surveillance regimen including lifelong cystoscopy.

From the Department of Surgery (Urology), McGill University, Montréal, QC

This paper has been peer-reviewed.

Competing interests: None declared.

\section{References}

1. Marrett LD, De P, Dryer D. Steering Committee of Canadian Cancer Statistics 2008. CMAJ 2008;179: $1163-70$.

2. Brennan $P$, Bogillot 0 , Cordier $S$, et al. Cigarette smoking and bladder cancer in men: a pooled analysis of 11 case-control studies. Int I Cancer 2000;86:289-94.

3. Pashos CL, Botteman MF, Laskin BL, et al. Bladder cancer: epidemiology, diagnosis and management Cancer Pract 2002;10:311-22.

4. Castelao JE, Yuan JM, Gago-Dominguez $M$, et al. Non-steroidal antiinflammatory drugs and bladder cancer prevention. Br J Cancer 2000;82:1364-9.

5. Chrouser K, Leibovich B, Bergsitalh E, et al. Bladder cancer risk following primary and adjuvant externa beam radiation for prostate cancer. J Urol 2006;174:107-10.

6. Steinmaus $C M$, Nuñez $S$, Smith AH. Diet and bladder cancer: a meta-analysis of six dietary variables. Am J Epidemiol 2000;151:693-702.

7. Schistosomes, liver flukes and Helicobacter Pylori. IARC Working Group on the Evaluation of Carcinogenic Risks to Humans. Lyon, 7-14 June, 1994. IARC Monogr Eval Carcinog Risks Hum 1994;61:1-241.

8. Travis $L B$, Curtis RE, Glimelius B, et al. Bladder and kidney cancer following cyclophosphamide therapy for non-Hodgkin's lymphoma. J Nat Cancer Inst 1995;87:524-30.

9. Madersbacher $S$, Hochreiter $W$, Burkhard $F$, et al. radical cystectomy for bladder cancer todayhomogeneous series without neoadjuvant therapy. I Clin Oncol 2003;4:690-6.

10. Ghoneim MA, Abdel-Latif $M$, El-Mekresh $M$, et al. Radical cystectomy for carcinoma of the bladder: 2,720 consecutive cases 5 years later. J Urol 2008;180:121-7.

11. Stein JP, Lieskovsky $G$, Cote R, et al. Radical cystectomy in the treatment of invasive bladder cancer: long-term results in 1,054 patients. J Clin Oncol 2001;19:666-75.
12. World Health Organization (WHO) Consensus Conference on Bladder Cancer; Hautmann R, Abol-Enein H, Hafez K, et al. Urinary diversion. Urology 2007;69:17-49.

13. Kessler T. Attempted nerve-sparing surgery and age have a significant effect on urinary continence and erectile function after radical cystoprostatectomy and ileal orthotopic substitution. J Urol 2004; 172:1323-7.

14. Shipley WU, Kaufman DS, Zehr E, et al. Selective bladder preservation by combined modality protocol treatment: long-term outcomes of 190 patients with invasive bladder cancer. Urology 2002;60:62-7.

15. Skinner DG. The value of regional lymph node dissection in genitourinary cancer. Semin Surg Oncol 1989;5:235-9.

16. Dhar NB, Klein EA, Reuther AM, et al. Outcome after radical cystectomy with limited or extended pelvic lymph node dissection. J Urol 2008;179:873-8.

17. Kassouf W, Aqarwal PK, Herr HW, et al. Lymph node density is superior to TNM nodal status in predicting disease-specific survival after radical cystectomy for bladder cancer: analysis of pooled data from MDACC and MSKCC. J Clin Oncol 2008;26:121-6.

18. Kassouf W, Leibovici D, Munsell MF, et al. Evaluation of the relevance of lymph node density in a contemporary series of patients undergoing radical cystectomy. J Urol 2006;176:53-7.

19. Herr HW, Bochner BH, Dalbagni $G$, et al. Impact of the number of lymph nodes retrieved on outcome in patients with muscle invasive bladder cancer. J Urol 2002;167:1295-8.

20. Herr HW, Faulkner JR, Grossman HB, et al. Surgical factors influence bladder cancer outcomes: a cooperative group report. J Clin Oncol 2004;22:2781-9.

21. Coen JJ, Zietman AL, Kaufman DS, et al. Benchmarks achieved in the delivery of radiation therapy for muscle-invasive bladder cancer. Urol Oncol 2007;25:76-84.

22. Freiha F, Reese J, Torti FM. A randomized trial of radical cystectomy v. radical cystectomy plus cisplatin, vinblastine and methotrexate chemotherapy for muscle invasive bladder cancer. J Urol 1996;155:495-9.

23. Stockle M, Meyenburg W, Wellek S, et al. Advanced bladder cancer (stages pT3b, pT4a, pN1 and pN2): improved survival after radical cystectomy and 3 adjuvant cycles of chemotherapy. Results of a controlled prospective study. J Urol 1992;148:302-7.

24. Skinner DG, Daniels JR, Russell CA, et al. The role of adjuvant chemotherapy following cystectomy for invasive bladder cancer: a prospective comparative trial. J Urol 1991;145:459-64.

25. Adjuvant chemotherapy in invasive bladder cancer: a systematic review and meta-analyis of individual patient data; Advanced Bladder Cancer (ABC) Meta-analysis Collaboration. Eur Urol 2005;48:189-99.

26. Shariat SF, Karakiewicz PI, Palapattu GS, et al. Outcomes of radical cystectomy for transitional cell carcinoma of the bladder: a contemporary series from the Bladder Cancer Research Consortium. J Urol 2006; 176:2414-22

27. Milosevic M, Gospodarowicz M, Zietman AL, et al. Radiotherapy for bladder cancer. Urology 2007;69: 80-92.

28. Kachnic LA, Kaufman DS, Heney NM, et al. Bladder preservation by combined modality therapy for invasive bladder cancer. J Clin Oncol 1997;15:1022-9.

29. Shipley WU, Kaufman DS, Heney NM, et al. An update of selective bladder preservation by combined modality therapy for invasive bladder cancer. Eur Urol 1998;33:32-4.

30. Sauer R, Birkenhake $S$, Kuhn R, et al. Efficacy of radiochemotherapy with platin derivatives compared to radiotherapy alone in organ-sparing treatment of bladder cancer. Int J Radiat Oncol Biol Phys 1998;40: 121-7.

31. Housset $M$, Maulard $C$, Chretien $Y$, et al. Combined radiation and chemotherapy for invasive transitional cell carcinoma of the bladder: a prospective study. J Clin Oncol 1993;11:2150-7.

32. Dunst J, Saver R, Schrott KM, et al. Organ-sparing treatment of advanced bladder cancer: a 10-year experience. Int J Radiat Oncol Biol Phys 1994;30:261-6.

33. Hautmann RE, de Petriconi R, Volkmer BG. Neobladder formation after pelvic irradiation. World J Urol 2009;27:57-62.

34. Hautmann RE. Complications and results after cystectomy in male and female patients with locally invasive bladder cancer. Eur Urol 1998; 33: 23-4.

35. Bochner BH, Figueroa AJ, Skinner EC, et al. Salvage radical cystoprostatectomy and orthotopic urinary diversion following radiation failure. J Urol 1998;160:29-33.

36. Gheiler EL, Wood DP Jr, Montie JE, et al. Orthotopic urinary diversion is a viable option in patients undergoing salvage cystoprostatectomy for recurrence prostate cancer after definitive radiation therapy. Urology 1997;50:580-4.

37. Nieder AM, Porter MP, Soloway MS. Radiation therapy for prostate cancer increases subsequent risk of bladder and rectal cancer: a population based cohort study. I Urol 2008;180:2005-9.

38. Wolf $\mathrm{H}, \mathrm{Olsen}$ PR, Hoigaard K. Urothelial dysplasia concomitant with bladder tumours: a determinant for future new occurrences in patients treated by full-course radiotherapy. Lancet 1985;1:1005-8.

39. Babjuk M, Oosterlinck W, Sylvester R, et al. EUA guidelines on non-muscle-invasive urothelial carcinoma of the bladder. Eur Urol 2008:54:303-14. 
40. Vaidya A, Soloway MS, Hawke C, et al. De novo muscle-invasive bladder cancer: Is there a change in trend? J Urol 2001;165:47-50.

41. Herr HW, Sogani PC. Does early cystectomy improve the survival of patients with high risk superficial bladder tumours? J Urol 2001;166:1296-9.

42. Harland SJ, Kynaston H, Grigor K, et al. A randomized trial of radical radiotherapy for the management of pTIG3 NXMO transitional cell carcinoma of the bladder. J Urol 2007;178:807-13.
Correspondence: Dr. Wassim Kassouf, MD, FRCSC, Division of Urology, McGill University Health Centre, 1650 Cedar Ave., Rm 18-315, Montréal, QC H3G 1A4; fax 514-934-8297; wassim.kassouf@muhc.mcgill.ca

\title{
In favour of bladder preservation using combined modality treatment
}

\author{
Himu Lukka, MBCLB, FRCP(UK), FRCR(UK), FRCPC
}

$\Delta$ II health care professionals would agree that the primary goal of bladder cancer treatment should be to maximize patient survival while minimizing toxicity and negative impact on a patient's quality of life (QOL). Bladder preservation (and its impact on QOL) can only be considered an important secondary objective. Bladder cancer management has seen a significant change with the increasing use of multimodality treatment (e.g., surgery, radiotherapy and/or chemotherapy) as opposed to unimodality treatment.

To enable all treatment options to be discussed and to avoid unnecessary delays in treatment, ideally patients should be seen by urologists, radiation oncologists and medical oncologists in a multidisciplinary setting. Urologists would be strongly encouraged to refer their patients with invasive bladder cancer for a multidisciplinary opinion with the understanding that patients requiring a cystectomy would be referred back to the referring urologists.

\section{Cystectomy as a treatment option}

Radical cystectomy remains the primary treatment in the local management of bladder cancer in North America. In contrast, bladder preservation with salvage cystectomy in Europe has had a longer history-in some centres, it is the recommended approach. In North America, the centre with the largest experience with bladder preservation using a trimodality treatment is Massachusetts General Hospital $(\mathrm{MGH})$ in Boston. While continent urinary tract reconstruction using cutaneous urinary reservoirs or orthotopic diversion is a step up from urinary diversion with an ileal conduit and urostoma, limited number of patients, in practice, have continent urinary tract reconstruction.

\section{Results of cystectomy}

Two large recent radical cystectomy series provide the best data outcomes (Table 1). The University of Southern California (USC) series of 633 patients with pT2-T4a reported 5 and 10 years overall survival of $48 \%$ and $32 \% .^{1}$ The Memorial Sloan Kettering Cancer Center (MSKCC) series of 184 patients with pT2-4 reported overall 5-year survival rates of $36 \% .^{2}$ While the operative and perioperative care has improved, operative mortality in modern series ranges from $1 \%$ to $2 \%$ and postoperative complications range from $15 \%$ to $32 \%{ }^{3,4}$ Studies confirm that the most serious side effects are urinary diversion and loss of sexual function in men and women; these effects also have a significant impact on QOL. Continent urinary procedures achieve $82 \%$ continence rates, yet complications require reoperation in $10 \%$ to $15 \%$ of these patients.

\section{Bladder preservation}

Radiotherapy in the local management of bladder cancer has seen a significant change over the last couple of decades, evolving from the use of radiotherapy alone to preoperative radiotherapy, and more recently the use of trimodality treatment. The modern approach to bladder preservation involves careful cystoscopic evaluation, transurethral resection of the bladder to minimize the extent of residual disease, concurrent chemoradiotherapy followed by regular cystoscopic evaluation, and salvage cystectomy for recurrence. These strategies require the active participation of the urologist in the preradiotherapy assessment, decisionmaking, postradiotherapy monitoring and surgical intervention for salvage radiotherapy.

Concurrent cisplatinum with radiotherapy is recommended to improve local control based on the National Cancer Institute 